\title{
Differentiable Functions Equivalent to Analytic Functions
}

By

\author{
Masahiro ShIota
}

1. Let $f, g$ be real-valued functions of class $C^{\infty}$ in $\mathbf{R}^{1}$. Functions $f, g$ are called equivalent if there exists a diffeomorphism (of class $C^{\infty}$ ) $\tau$ of $\mathbf{R}^{1}$ such that $f \circ \tau=g$. The main object of this paper is to show under what conditions a function is equivalent to an analytic function (Theorem 1).

In the case of polynomials, the corresponding result is proved in Thom [1]. The method of our proof is analogous to that in [1], and our Lemma 3,4 correspond to Theorem $R$ in [1].

Theorem 2 refines Mittag-Lefler's theorem in the real case.

The author thanks Mr. Iwasaki for his kind criticisms, and Professor S. Matsuura for his kind encouragement.

2. A function is called flat at a point $a$ if for each $n \geqq 0$ the $n$-th derived function $f^{(n)}$ vanishes at $a$.

Theorem 1. A $C^{\infty}$-function $f$ (not constant) is equivalent to an analytic function if and only if the derived function $f^{\prime}$ is nowhere flat.

If $f^{\prime}$ is nowhere flat, then we can see by Rolle's theorem that the set of critical points of $f$ (i.e. the set of points where $f^{\prime}$ vanishes) has no accumulating points. Let $\left\{a_{n}\right\}$ denote the set. Adding regular points to the set (if necessary), we can assume $\left\{a_{n}\right\}$ satisfies the following conditions

(1) $a_{n}<a_{n+1}$

(2) $a_{n} \rightarrow \infty(n \rightarrow \infty) \quad a_{n} \rightarrow-\infty(n \rightarrow-\infty)$,

(3) $a_{-1}<0<a_{0}$.

For each integer $n$ we define $k(f, n)$ the least non-negative integer $k-1$ 
such that the $k$-th derived function $f^{(k)}$ does not vanish at $a_{n}$, and put

$$
b(f, n)= \begin{cases}(-1)^{\sum_{l=0}^{n} k(f, l)}\left(f\left(a_{n+1}\right)-f\left(a_{n}\right)\right) & n \geqq 0 \\ f\left(a_{0}\right)-f\left(a_{-1}\right) & n=-1 \\ (-1)^{l_{l=n+1}^{-1} k(f, l)}\left(f\left(a_{n+1}\right)-f\left(a_{n}\right)\right) & n \leqq-2 .\end{cases}
$$

Then $b(f, n)$ have all the same sign.

For the proof we need the following lemmas.

Lemma 1. Let $c, d$ be real numbers $(0<c<d)$ and $h$ a real-valued continuous function in $(-\infty, c] \cup[d, \infty)$. Then there is an entire holomorphic function $\phi$ in the complex plane $C^{1}$ which satisfies the following conditions:

(1) the restriction of $\phi$ on the real axis is real-valued,

(2) $\phi(x) \leqq h(x)$ on $(-\infty, c] \cup[d, \infty)$,

$$
\phi(x) \geqq 0 \quad \text { on }\left[\frac{2}{3} c+\frac{1}{3} d, \frac{1}{3} c+\frac{2}{3} d\right] \text {, }
$$

(3) $\operatorname{Re} \phi(z) \leqq h(c)$ on $|z| \leqq c$.

Proof. It is enough to prove the lemma for a function $h^{\prime}$ such that $h^{\prime} \leqq h$. So, from the first we can assume

$$
h=\left\{\begin{array}{lll}
K_{n}+1 & \text { on }[n d,(n+1) d) & \text { for } n \neq 0,-1\left(K_{n}<0\right), \\
K+1 & \text { on }[-d, c] & (K<0) .
\end{array}\right.
$$

We put

$$
\begin{aligned}
& \phi_{0}(z)=\frac{-K+1}{d-c}(3 z-2 c-d)+1, \\
& \phi_{n}(z)=K_{n} e^{l_{n}\{-z+(n+1) d\}} \quad n \leqq-2,
\end{aligned}
$$

$\left(l_{n}\right.$ are taken large enough so that $\left|\phi_{n}(z)\right| \leqq 2^{-n}$ on $\left.|z| \leqq \max \left(\frac{-n-1}{2} d, c\right)\right)$

$$
\phi_{n}(z)=\left(K_{n}+\frac{K-1}{d-c}(3 n d+2 d-2 c)\right) e^{l_{n}(z-n d)} \quad n \geqq 1,
$$


( $l_{n}$ are taken large enough so that $\left|\phi_{n}(z)\right| \leqq 2^{-n}$ on $|z| \leqq \max \left(\frac{n d}{2}, c\right)$ ). Then for any compact set $K\left(\subset \mathbb{C}^{1}\right), \sum_{n \neq-1} \phi_{n}$ converges uniformly on $K$, so $\phi=\sum_{n \neq-1} \phi_{n}$ is holomorphic. It is easily seen that $\phi$ satisfies the conditions in the lemma.

Lemma 2. Let $c, d$ be real numbers $(0<c<d)$ and $h$ a positive continuous function in $(-\infty, c] \cup\left[\frac{2}{3} c+\frac{1}{3} d, \frac{1}{3} c+\frac{2}{3} d\right] \cup[d, \infty)$. Then there is an entire holomorphic function $\phi$ in $\mathbf{C}^{1}$ which satisfies the following conditions.

(1) the restriction of $\phi$ on the real axis is real-and positive-valued,

(2) $\phi(x) \leqq h(x)$ on $(-\infty, c] \cup[d, \infty)$,

$$
\phi(x) \geqq h(x) \text { on }\left[\frac{2}{3} c+\frac{1}{3} d, \frac{1}{3} c+\frac{2}{3} d\right] \text {, }
$$

(3) $|\phi(z)| \leqq h(c)$ on $|z| \leqq c$.

Proof. Applying Lemma 1 to

$$
\log h(x)-\log \underset{x \in\left[-\frac{2}{3}-c+\frac{1}{3} d, \frac{c}{3}+-_{3}^{2}-d\right]}{\sup },
$$

we easily prove this lemma.

Lemma 3. For any real numbers $a_{n} \neq 0$ (such that $a_{n}<a_{n+1}, a_{n} \rightarrow$ $\infty(n \rightarrow \infty)$, and $\left.a_{n} \rightarrow-\infty(n \rightarrow-\infty)\right)$, non-negative integers $k_{n}$, and postive numbers $b_{n}$, there is an entire holomorphic function $g$ in $\mathbb{C}^{1}$ which satisfies the following conditions.

(0) the restriction of $g$ on the real axis is real-valued,

(1) the set of critical points of $g$ in the real axis is contained in the sequence $\left\{a_{n}\right\}$,

(2) $k(g, n)=k_{n}$ where $k(g, n)$ means $k(g \mid \mathbf{R}, n)$,

(3) $0<b(g, n) \leqq b_{n}$ for $n \neq 0$

$$
b(g, 0)=b_{0} \text { for } n=0,
$$

where $b(g, n)$ means $b(g \mid \mathbf{R}, n)$.

Proof. We put

$$
H_{n}(z)=\exp \left[k_{n}\left\{\left(\frac{z}{a_{n}}\right)+\frac{1}{2}\left(\frac{z}{a_{n}}\right)^{2}+\cdots+\frac{1}{|n|}\left(\frac{z}{a_{n}}\right)^{|n|}\right\}\right] \text {, for each } n
$$




$$
G(x)=\int_{0}^{x} \prod_{n=-\infty}^{\infty}\left(1-\frac{z}{a_{n}}\right)^{k_{n}} H_{n}(z) d z
$$

then by Mittag-Lefler's theorem $G(x)$ is an entire holomorphic function in $\mathbf{C}^{1}$ which satisfies the conditions (1), (2) in the lemma. Let $h$ be a positive valued continuous function such that

small enough on $\left(-\infty, a_{0}\right] \cup\left[a_{1}, \infty\right)$,

large enough on $\left[\frac{2}{3} a_{0}+\frac{1}{3} a_{1}, \frac{1}{3} a_{0}+\frac{2}{3} a_{1}\right]$.

If we apply Lemma 2 to this $h$, then we get an entire holomorphic function $\phi(z)$ such that

$$
g(x)=\delta \int_{0}^{x} \phi(z) \prod_{n=-\infty}^{\infty}\left(1-\frac{z}{a_{n}}\right)^{k_{n}} H_{n}(z) d z \quad(\delta \text { is a constant })
$$

satisfies the conditions (1), (2), (3) in the lemma.

Lemma 4. In Lemma 3, we can take $g$ so as to satisfy moreover the following condition,

$$
b(g, n)=b_{n} \quad \text { for each } n .
$$

Proof. For each $m$ we have constructed an entire holomorphic function $g_{m}$ in $\mathbf{C}^{1}$ which verifies (1), (2) in Lemma 3 and a condition

$$
\begin{array}{ccrl}
0<b\left(g_{m}, n\right) & \leqq 2^{-|m|-3} b_{n} & \text { for } & m \neq n, \\
b\left(g_{m}, n\right) & =b_{n} & \text { for } & m=n .
\end{array}
$$

In doing this, if we let $h$ take values small enough at $a_{m}$ and $a_{m+1}$, from the condition 3 in Lemma $2 g_{m}$ is constructed so that for any compact set $K\left(\subset \mathbf{C}^{1}\right) \sum c_{m} g_{m}$ (for any $0<c_{m} \leqq 1$ ) converges uniformly on $K$. Here we should remark that $\sum c_{m} g_{m}\left(0<c_{m} \leqq 1\right)$ satisfies the (1), (2) in Lemma 3 . We put

$$
\begin{aligned}
& g_{1}^{\circ}=\sum_{m=-\infty}^{\infty} g_{m}, \text { then } 1<\frac{b\left(g_{1}^{\circ}, n\right)}{b_{n}}<\frac{3}{2} \\
& g_{2}^{\circ}=g_{1}^{\circ}+\sum_{m=-\infty}^{\infty} c_{m, 1} g_{m},\left(c_{m, 1}=\sup _{n} \frac{b\left(g_{1}^{\circ}, n\right)}{b_{n}}-\frac{b\left(g_{1}^{\circ}, m\right)}{b_{m}}\right)
\end{aligned}
$$

then 


$$
\sup _{m} \frac{b\left(g_{1}^{\circ}, m\right)}{b_{m}}<\frac{b\left(g_{2}^{\circ}, n\right)}{b_{n}}<\frac{3}{2} \sup _{m} \frac{b\left(g_{1}^{\circ}, m\right)}{b_{m}}-\frac{1}{2}
$$

generally we put

$$
\begin{aligned}
g_{k}^{\circ}=g_{k-1}^{\circ}+\sum_{m=-\infty}^{\infty} c_{m, k-1} g_{m}, \\
\quad\left(c_{m, k-1}=\sup _{n} \frac{b\left(g_{k-1}^{\circ}, n\right)}{b_{n}}-\frac{b\left(g_{k-1}^{\circ}, m\right)}{b_{m}}\right)
\end{aligned}
$$

then

$$
\sup _{m} \frac{b\left(g_{k-1}^{\circ}, m\right)}{b_{m}}<\frac{b\left(g_{k}^{\circ}, n\right)}{b_{n}}<\frac{3}{2} \sup _{m} \frac{b\left(g_{k-1}^{\circ}, m\right)}{b_{m}}-\frac{1}{2} \sup _{m} \frac{b\left(g_{k-2}^{\circ}, m\right)}{b_{m}} .
$$

From this we can see $0 \leqq c_{m, k} \leqq\left(\frac{1}{2}\right)^{k}$. If we put

$$
\begin{gathered}
c_{m}=1+\sum_{k=1}^{\infty} c_{m, k}, \\
g^{\circ}=\sum_{m=-\infty}^{\infty} c_{m} g_{m} .
\end{gathered}
$$

Then $g^{\circ}$ has the property

$$
\frac{b\left(g^{\circ}, n\right)}{b_{n}}=\frac{b\left(g^{\circ}, m\right)}{b_{m}}, \text { for any } n, m .
$$

So $g=\frac{b_{n}}{b\left(g^{\circ}, n\right)} g^{\circ}$ satisfies the condition (4) in the lemma.

Proof of Theorem 1. The necessity of the condition is trivial. We shall prove its sufficiency. From Lemma 3, 4 there is an analytic function $g$ in $\mathbf{R}^{1}$ such that

(1) the set of critical points of $g$ is contained in the sequence $\left\{a_{n}\right\}$,

(2) $k(g, n)=k(f, n)$,

(3) $b(g, n)=b(f, n)$,

(4) (by adding a constant) $g\left(a_{n}\right)=f\left(a_{n}\right)$.

Let $\tau$ be a function in $\mathbf{R}^{1}$ such that

$$
\tau(x)=f^{-1} g(x) \cap\left[a_{n}, a_{n+1}\right] \text { on }\left[a_{n}, a_{n+1}\right] .
$$

Then $\tau$ is diffeomorphic on $\left(a_{n}, a_{n+1}\right)$. Around $a_{n}$ there are certain $C^{\infty}$ - 
functions $F, G$ such that

$$
\begin{aligned}
& f(x)=\delta\left\{\left(x-a_{n}\right) F(x)\right\}^{k(f, n)+1}+f\left(a_{n}\right),\left(\delta=\text { a constant, } F\left(a_{n}\right)>0\right) \\
& g(x)=\delta\left\{\left(x-a_{n}\right) G(x)\right\}^{k(f, n)+1}+f\left(a_{n}\right) .\left(G\left(a_{n}\right)>0\right)
\end{aligned}
$$

From this, $\tau$ is locally diffeomorphic around $a_{n}$. So $\tau$ is a diffeomorphism of $\mathbf{R}^{1}$, and satisfies $f \circ \tau=g$.

\section{Applications of the lemmas}

Next lemmas result from the corresponding previous lemmas and proofs.

Lemma 2'. Let $c, d$, e be real numbers $(0<c<d, 0<e) h$ a positivevalued continuous function $(h(x)>e$ on $[c, d])$. Then there is an entire holomorphic function $\phi$ in $\mathbf{C}^{1}$ which satisfies the following conditions

(1) the restriction of $\phi$ on the real axis is real-and positive-valued,

(2) $\phi(x) \leqq h(x)$ on the real axis,

(3) $\phi(x) \geqq e$ on $[c, d]$,

(4) $|\phi(z)| \leqq h\left(\frac{c}{2}\right)$ on $|z| \leqq \frac{c}{2}$.

Lemma $2^{\prime \prime}$. Let $c, d, e, h$ be the same ones as in Lemma $2^{\prime}$. Let $\left\{a_{n}\right\},\left\{b_{n}^{\prime}\right\}$ be sets of real numbers which satisfy

(a) $a_{n}<a_{n+1}, a_{-1}<0<a_{0}, a_{n} \rightarrow \infty(n \rightarrow \infty), a_{n} \rightarrow-\infty(n \rightarrow-\infty)$,

(b) $0<b_{n}^{\prime}<h\left(a_{n}\right)$ when $a_{0} \geqq c$ and $-a_{-1} \geqq c$, the set of numbers $\frac{b_{n}^{\prime}}{h\left(a_{n}\right)}$ is bounded, otherwise,

(c) $\left\{a_{n}\right\} \cap[c, d]=\phi$.

Then, there are an entire holomorphic function $\phi$ in $\mathbb{C}^{1}$ and a constant $\delta$ $(>0)$ which satisfy the condition (1), (2), (3), (4) in Lemma 2', and a condition

(5) $\phi\left(a_{n}\right)=b_{n}^{\prime}$ when $a_{0} \geqq c$ and $-a_{-1} \geqq c$,

$$
\phi\left(a_{n}\right)=\delta b_{n}^{\prime} \text { otherwise. }
$$

Lemma 4'. For positive numbers $c_{n}$, the $g$ (in Lemma 4) can be 
chosen to satisfy moreover

$$
g^{\left(k_{n}+1\right)}\left(a_{n}\right)=\left\{\begin{array}{lll}
(-1)^{\sum_{i=0}^{n} k_{i}} c_{n} & n \geqq 0, \\
& & n=-1, \\
c_{-1} & & \\
(-1)^{\sum_{i=n+1}^{-1} k_{i}} c_{n} & n \leqq-2 .
\end{array}\right.
$$

Lemma $4^{\prime \prime}$. On the same conditions as in Lemma $4^{\prime}$, for any $\delta>0$ there are entire holomorphic functions $g_{N, \varepsilon}(z)$ in $\mathbf{C}^{1}(N$ : positive integer $0<\varepsilon \leqq \varepsilon(N)$ where $\varepsilon(N)>0$ is defined on positive integers) which satisfy the conditions (1), (2) in Lemma 3 and the following conditions

(i) $\quad g_{N, \varepsilon}^{\left(k_{n}+1\right)}\left(a_{n}\right)=\left\{\begin{array}{cc}(-1)^{\sum_{i=0}^{n} k_{i}} c_{n} & n \geqq N+1 \\ (-1)^{i=n+1} c_{n}^{-1} c_{i} & n \leqq-N+1,\end{array}\right.$

(ii) $b\left(g_{N, \varepsilon}, n\right)= \begin{cases}b_{n} & |n| \geqq N \\ \varepsilon b_{n} & |n|<N,\end{cases}$

(iii) $\left|g_{N, \varepsilon}(z)\right|<\delta \quad|z|<\frac{\min \left(a_{N},-a_{-N}\right)}{2}$.

Theorem 2. Let $\left\{a_{n}\right\},\left\{l_{n}\right\},\left\{c_{n}\right\}$ be sets of real numbers which satisfy

(a) $\{n\}=\{$ integer $\}$, or $\{n \geqq N\}$ for some $N$, or $\{n \leqq N\}$ for some $N$,

(b) $a_{n}<a_{n+1}, a_{n} \rightarrow \infty$ (as $\left.n \rightarrow \infty\right), a_{n} \rightarrow-\infty$ (as $\left.n \rightarrow-\infty\right)$,

(c) $l_{n}$ are positive integers,

(d) $c_{0},(-1)^{\sum_{i=1}^{n} l_{i}} c_{n}(n>0),(-1)^{-\sum_{i=n+1}^{-1} l_{i}} c_{n}(n<0)$

have the same sign.

Then there is an analytic function $f$ in $\mathbf{R}^{1}$ such that

(i) the set of zero points of $f$ is $\left\{a_{n}\right\}$,

(ii) for each $n, a_{n}$ is a zero point of $l_{n}$-th order of $f$, 
(iii) for each $n, f^{\left(l_{n}\right)}\left(a_{n}\right)=c_{n}$.

Proof. We assume $\{n\}=\{$ integer $\}$, otherwise we can prove in a similar way. If $c_{-1}$ is positive, then we prove about $\left\{-c_{n}\right\}$. So we assume $c_{-1}$ is negative, and $a_{-1}<0<a_{0}$. We put

$$
\begin{aligned}
& a_{2 n}^{\prime}=a_{n}, a_{2 n+1}^{\prime}=\frac{a_{n}+a_{n+1}}{2} \\
& k_{2 n}=l_{n}-1, k_{2 n+1}=1, \\
& b_{n}=1 \\
& c_{2 n}^{\prime}= \begin{cases}-(-1)^{\sum_{i=0}^{n} l_{\imath}} c_{n} & n \geqq 0 \\
-c_{1} & n=-1 \\
-(-1)^{\sum_{i=n+1}^{-1} l_{i}} c_{n} & n \leqq-2,\end{cases} \\
& c_{2 n+1}^{\prime}=1,
\end{aligned}
$$

from the above assumption, $c_{n}^{\prime}$ are positive. If we apply Lemma $4^{\prime}$ to $\left\{a_{n}^{\prime}\right\},\left\{k_{n}\right\},\left\{c_{n}^{\prime}\right\}$, then there is an analytic function $g$ in $\mathbf{R}^{1}$ such that

(1) the set of critical points of $g$ is contained in the sequence $\left\{a_{n}\right\}$,

(2) $k(g, n)=k_{n}$,

(3) $b(g, n)=1$,

(4) $g^{\left(k_{n}+1\right)}\left(a_{n}^{\prime}\right)= \begin{cases}(-1)^{\sum_{i=0}^{n} k_{i}} c_{n}^{\prime} & n \geqq 0 \\ c_{-1}^{\prime} & n=-1 \\ (-1)^{i=\sum^{-1} k_{i}} c_{n}^{\prime} & n \leqq-2 .\end{cases}$

From this it is easily seen that $g^{\left(l_{n}\right)}\left(a_{n}\right)=c_{n}, g\left(a_{2 n+2}^{\prime}\right)=g\left(a_{2 n}^{\prime}\right)$ and $g$ is a monotone function on $\left[a_{n}^{\prime}, a_{n+1}^{\prime}\right]$. If we put $f=g-g\left(a_{0}\right)$ then the set of zero points of $f$ is $\left\{a_{2 n}^{\prime}\right\}=\left\{a_{n}\right\}$, for each $n a_{n}$ is a zero point of $l_{n}$-th order of $f$, and for each $n f^{\left(l_{n}\right)}\left(a_{n}\right)=c_{n}$. 
Theorem 3. Let $\left\{a_{n}\right\}$ be a set of real numbers (such that $a_{n}<a_{n+1}$, $\left.a_{n} \rightarrow \infty(n \rightarrow \infty), a_{n} \rightarrow-\infty(n \rightarrow-\infty)\right)\left\{l_{n}\right\}$ be a set of positive integers, and $\left\{p_{n}(x)\right\}$ be a set of polynomials (such that for each $n$ the degree of $p_{n}(x)$ is less than $\left.l_{n}\right)$. Then there is an analytic function $f$ in $\mathbf{R}^{1}$ such that for each $n, a_{n}$ is a zero point of $l_{n}$-th order of $f(x)-p_{n}(x)$.

Proof. In the same way as the proof of Theorem 2, if we define adequate values of derivatives (of the function which we want) at each point $\frac{a_{n}+a_{n+1}}{2}$, then we get an entire holomorphic function $f_{0}$ in $\mathbf{C}^{1}$ such that for each $n f_{0}\left(a_{n}\right)=p_{n}\left(a_{n}\right)$. We put

$$
\begin{aligned}
p_{n, 1}(x)=p_{n}(x)-\left\{f_{0}\left(a_{n}\right)\right. & +\left(x-a_{n}\right) f_{0}^{\prime}\left(a_{n}\right)+\cdots \\
& \left.+\left(x-a_{n}\right)^{l_{n}}\left(l_{n} !\right)^{-1} f_{0}^{\left(l_{n}\right)}\left(a_{n}\right)\right\}
\end{aligned}
$$

and defining adequate values of derivatives (of the function which we want) at one or two points of each $\left(a_{n}, a_{n+1}\right)$, we get an entire holomorphic function $f_{1}$ in $\mathbf{C}^{1}$ such that

$$
\begin{aligned}
& f_{1}\left(a_{n}\right)=p_{n, 1}\left(a_{n}\right)=0, \\
& f_{1}^{\prime}\left(a_{n}\right)=p_{n, 1}^{\prime}\left(a_{n}\right) .
\end{aligned}
$$

Repeating this and applying Lemma 4 ", we get entire holomorphic functions $f_{m}$ and polynomials $p_{n, m}$ which satisfy the following conditions

(1) $f_{n}^{(p)}\left(a_{n}\right)=p_{n, m}^{(p)}\left(a_{n}\right) \quad$ for $\quad p \leqq m$,

(2) $\quad p_{n, m}(x)=p_{n, m-1}(x)-\left\{f_{m-1}\left(a_{n}\right)+\cdots+\left(x-a_{n}\right)^{l_{n}}\left(l_{n} !\right)^{-1} f_{m-1}^{\left(l_{n}\right)}\left(a_{n}\right)\right\}$

(3) for any compact set $K\left(\subset \mathbf{C}^{1}\right) \sum f_{n}$ converges uniformly on $K$.

The function $\sum f_{n}$ is what we want.

\section{Reference}

[1] Thom, R., L'équivalence d'une fonction différentiable et d'un polynome, Topology 3. Suppl. 2 (1965), 297-307.

Department of Mathematics Kyoto University. 
